Aus der Greifswalder Chirurgischen Universitätsklinik. (Direktor : Geheimrat Prof. Dr. Pels-Leusden.)

\title{
Über die Behandlung der pyogenen Blutinfektion durch intravenöse Anwendung von Urotropin.
}

\author{
Von Dr. med. Arthur Buzello, Assistent.
}

Trotz der sehr großen Menge der heute gebräuchlichen Behandlungsmethoden der pyogenen Blutinfektion ist die Prognose bei schweren Fällen mit positivem Bakterienbef und im Blut immer noch äußerst ungünstig. Wenn auch auf diesem Krankheitsgebiet zahlenmäßige Angaben nicht viel bedeuten, weil der Krankheitsbegriff noch nicht fest begrenzt und scharf charakterisiert ist, so läßt sich doch sagen, daß die Angaben von Lenhartz, der in seinem „klassischen" Werke über die septischen Erkrankungen unter 77 Fällen septischer Allgemeininfektion mit positivem Bakterienbefund im Blut eine Mortalität von 83 Proz. findet, auch bei der weit größeren Zahl der heute angewandten spezifischen Mittel sich nur unbedeutend zum Besseren verändert haben. Es gehen heute in den Zahlenangaben mehr Fälle in Heilung aus; das beruht aber nur zum Teil auf der breiteren und erfolgreichen Anwendung therapeutischer Mittel, zum großen Teil ist diese Veränderung aber auch darauf zurückzuführen, daß mit der Verbesserung und häufigeren Anwendung der bakteriologischen Blutuntersuchung die Krankheit jetzt häufiger diagnostiziert wird als früher. Naturgemäß gibt es auch bei dieser Krankheit leichte und schwere Fälle, je nachdem, ob ein gesunder oder ein durch langes Krankenlager erschöpfter Körper sekundär von der Infektion befallen wird. Es braucht nicht jede pyogene Blutinfektion zu Metastasen in fast allen inneren Organen und zum Tode zu führen. Verhältnismäßig günstig wird immer die Prognose der chirurgischen pyogenen Erkrankungen mit Blutinfektion sein, da in einer großen Anzahl der Fälle die radikale Beseitigung des 
Ausgangsherdes der Infektion sich ermöglichen läßt und damit der weiteren Blutinfektion der Boden entzogen wird., Aber auch hier sind die Zahlenangaben noch sehr verschieden. Bertels. $\mathrm{m}$ a $\mathrm{n}$ hat bei 48 chirurgischen pyogenen Blutinfektionen 42 Proz. geheiltc Fälle, Kiralyfi hat bei to Fällen nur 2 Todesfälle aufzuweisen, Brawermann dagegen unter 2 I Fällen nur 2 Heilungen. Die Staphylokokken-Blutinfektion hat nach Jochman $n$ im allgemeinen eine sehr ungünstige Prognose, noch ungünstiger allerdings die Infektion durch den Streptococcus longus. Die metastasierende Pneumokokkeninfektion endet fast ausnahmslos letal, nur die Blutinfektion durch den Gonorrhöecoccus und das Bacterium coli commune zeigen günstigeren Verlauf, bei letzterer berechnet Bondy 4I Proz. geheilte Fälle. Am ungünstigsten werden allgemein auch heute noch die puerperalen Blutinfektionen beurteilt, gleichviel, ob sie zu Metastasen führen oder nicht. Fast alle Autoren bezeichnen die Mortalität bei dieser letzten Form als über 8o Proz.

Gleich hier möchte ich den von mir gewählten Begriff der „pyogenen Blutinfektion" erläutern und rechtfertigen. Die Abgrenzung der Allgemeininfektion von der lokalen ist im Gesamtkrankheitsbilde oft schwierig. Örtliche pyogene Infektionen können sowohl durch ihre Ausdehnung als durch die Virulenz der Erreger oder durch das Befallen lebenswichtiger Organe zu schwersten Allgemeinerscheinungen führen, ohne daß wir berechtigt sind, von einer Allgemeininfektion des Körpers zu sprechen. Wie die örtliche pyogene Infektion durch Weiterverbreitung und phlegmonöses Wachstum zu immer neuen Infektionen im Körper den Anstoß gibt, die dann zu selbständigen Infektionsquellen werden, so ändert sich mit einem Schlage das Bild, sobald das lebenswichtigste Organ des Körpers, das Blut, von der Infektion ergriffen wird. Man könnte das reichlichere Eindringen der pyogenen Keime in die Blutbahn als eine schwere Metastase, als die gefährlichste Komplikation neben der Endocarditis, bezeichnen. Man kann schon aus rein klinischen Gesichtspunkten von der lokalen Infektion den Begriff der Blutinfektion, wenn diese letztere auch durch denselben pyogenen Erreger verursacht wird und am selben Körper auftritt, recht wohl abgrenzen. Es handelt sich dabei um zwei Krankheitsbilder, ätio- 
logisch in engster Beziehunng zueinander, verschieden durch die Art der Krankheitsäußerungen und ihre Wirkung auf den Gesamtorganismus. Wenn auch in der Mehrzahl der Fälle die Blutinfektion der lokalen Infektion folgt, so kann sie ihr auch vorangehen oder zum mindesten vor Ausbruch der örtlichen Erscheinungen bestehen, wie einwandfreie, wiederholte Untersuchungen dies beim Erysipel gezeigt haben (Koch, Weil, $\mathrm{Z}$ angemeister, Spassekukotzki), und wie ich dieses auch bei der Osteomyelitis fand, wenn es uns auch nur selten gelingen wird, die Anfänge der Infektion bakteriologisch festzulegen, weil die Patienten fast immer erst bei Ausbruch der örtlichen Infektion die klinische Behandlung aufsuchen. Auch sind durch die verbesserte Technik der bakteriologischen Blutuntersuchung wiederholt Beobachtungen bekannt geworden, bei denen sich in der Blutbahn andere Erreger nachweisen ließen als am Orte des lokalen Eiterungsprozesses, oder aber aus der Summe der Eiterung und Fäulnis erregenden Bakterien eines lokalen Prozesses nur eine einzelne bestimmte Art in die Blutbahn eingedrungen war und hier isoliert bis zum Tode verweilte. DaB die Blutinfek. tion in gewissem Sinne eine Krankheit für sich darstellt, zeigen die allgemein bekannten und charakteristischen Krankheitssymptome, wie Schüttelfrost, typische Temperaturanstiege, Erbrechen, septische Exantheme usw., die diese Infektion vor der örtlichen Infektion auszeichnen und immer den ganzen Charakter der Erkrankung durch ihr Hinzutreten wesentlich ernster gestalten. $\mathrm{Ob}$ man die so bekannten Krankheitsäußerungen der Infektion der Blutbahn im klinischen Gebrauch mit Sepsis, Pyämie, Bakteriämie, Septicopyämie, Toxinämie usw. bezeichnen soll, darüber ist zurzeit noch immer keine Einigung erzielt. Der Name ist an sich auch belanglos. Eine klinische Erfahrungstatsache ist jedoch, daß die vorher erwähnten charakteristischen Symptome nur durch reichliches Eindringen von pyogenen Erregern in die Blutbahn hervorgerufen werden können. Nur das gemeinsame Auftreten dieser Symptome, verbunden mit dem positiven bakteriologischen Nachweis zahlreicher Bakterienkolonien im Blut berechtigt zur Annahme einer pyogenen Blutinfektion. Es wird keinem Kliniker einfallen, einen sonst gesunden Menschen, bei dem sich im Rachenabstrich zufällig einige echte Diphtheriebazillen nach- 
weisen lassen, als einen Fall von Diphtheritis anzusehen und dementsprechend $z u$ behandeln, so wird auch niemand behaupten, daB ein Patient, der an einem Furunkel leidet und bei dem gelegentlich einzelne Bakterienkolonien im Blute nachweisbar. sind, an einer Blutinfektion leide. Nur der kulturelle Nachweis reichlicher Bakterien im Blut, verbunden mit den vorher beschriebenen charakteristischen klinischen Erscheinungen berechtigt zur Annahme einer pyogenen Blutinfektion. Ob dabei die erwähnten Krankheitssymptome als Abwehrreaktion des Körpers gegen die feindliche Invasion oder als Zeichen des Unterliegens der natürlichen Schutzkräfte des Organismus aufzufassen sind, ist bei dieser Betrachtung nicht entscheidend. Jedenfalls sind es Reaktionen des Körpers, die spezifisch sind für die Infektion der Blutbahn und die mit der Abwehr des Körpers gegen die örtliche Infektion nichts $z \mathfrak{u}$ tun haben. So glaube ich, daß man berechtigt ist, die Blutinfektion als besonderes Krankheitsbild aufzufassen und sie gesondert zu bekämpfen. Keinesfalls darf aber dieses besondere therapeutische Vorgehen die so häufige Ursache (oder auch Folge) derselben vernachlässigen, nämlich die primäre Eingangspforte der Erreger. Es muß daher immer ein Teil der Behandlung in der möglichst weitgehenden Unschädlichmachung des örtlichen Eiterungsprozesses bestehen. Im klinischen Gebrauch hat sich diese Trennung der Behandlung längst vollzogen, ohne daß man sich rein theoretisch davon Rechenschaft gab. Während die Bekämpfung der lokalen Eiterung nach allgemeinen Gesichtspunkten der Chirurgie als im allgemeinen feststehend und wenig veränderlich gilt, ist diese Einigkeit in der Behandlung der pyogenen Blutinfektion noch keineswegs erreicht. Ich will im folgenden nur von dieser letzteren Behandlung, also der der Blutinfektion als gesondertem Krankheitsbegriff, sprechen.

Schon aus der großen Anzahl der vorgeschlagenen Mittel und Methoden zur Bekämpfung der pyogenen Blutinfektion geht hervor, daß kein einziges dieser Mittel bisher die darauf gesetzten Hoffnungen ganz erfüllt hat. Wohl jede diesbezügliche Therapie hat ihre Erfolge und Mißerfolge zu verzeichnen. Es ist immer wieder der Gedanke, durch in die Blutbahn eingeführte artfremde Substanzen die darin enthaltenen pyogenen Keime restlos zu vernichten oder die im Körper vorhandenen Abwehrkräfte so zu 
stärken, daß der Organismus aus sich selbst heraus dieses Vernichtungswerk erfolgreich beenden kann. Noch scheint keine einzige Methode sich die unbedingte Anerkennung errungen zu haben. Fast immer findet man begeisterte Anhänger und auch die schärfsten Gegner einer neuen Methode. Oft wurden klinisch brauchbare Wege von bakteriologischer Seite vollständig verworfen, da den in die Blutbahn eingeführten Substanzen im Reagenzglasversuch jede bakterizide Wirkung abgesprochen werden mußte. Doch gerade bei der Blutinfektion zeigt es sich, vielleicht mehr wie in jedem andern Gebiete der Medizin, daß Reagenzglasversuche durchaus nicht beweisend sind für die Vorgänge in der Blutbahn, ja daß nicht einmal das Tierexperiment ausreicht, um sichere Schlüsse auf das Verhalten von artfremden Substanzen in der menschlichen Blutbahn zu ziehen. Den Grund zu letzterer geringen Beweiskraft des Experiments gibt sicherlich die Verschiedenheit der Pathogenität der Erreger für Mensch und Tier, dann die Verschiedenheit der Verhältnisse im Tierkörper und im menschlichen Organismus im gesunden und kranken $\mathrm{Zu}$ stand. Wohl kaum ein Therapeuticum ist so verschieden beurteilt worden wie das 1895 von Credé eingeführte Argentum colloidale (Collargol-Heyden). Die Zahl der Publikationen darüber beläuft sich heute bereits auf 600. Es hat begeisterte Anhänger gefunden (W i $B m a n n$ ). Andere, die seine Wirkung stark anzweifeln (Lenhartz, Kocher, Tavel, Kroenig. Rosthor $n, K$ üstner u. a.), und wieder andere, die es ganz ablehnen (Brickdale, Junk). Auch schwere Schädigungen teils örtlicher, teils allgemeiner Natur sind nach Anwendung des Collargols beschrieben worden ( $\mathrm{B}$ a um m, Cohn, B ondy). Ich selbst sah an der hiesigen Klinik eine schwere Gefäß- und Gewebsnekrose nach mehreren Collargolinjektionen in dieselbe Vene. Selbst die Biersche Stauungshyperämie, so ausgezeichnete Dienste sie bei der Behandlung lokaler Eiterungsprozesse leistet, ist gefährlich bei der Behandlung der Blutinfektion (D a u we, Stich, Lexer, Wrede, v. Graff). Wohl schon allgemein als aussichtslos erkannt und daher mit Recht verlassen ist heute die Antifermenttherapie (E. M üller, Kollarczeck, Jochmann). Wirklich überzeugende Erfolge hat auch die Serotherapie in der Behandlung der pyogenen Blutinfektion nicht gebracht. Ver- 
schiedene Mittel wurden angegeben, um im Blut eine künstliche Hyperleukocytose hervorzurufen, um die natürlichen bakteriziden Kräfte des Körpers zu stärken. Darauf hinzielend gaben Mik u licz und Miyake die Nucleïnsäure an, die ihre Anhänger in Hannes, Pollak und Hofbauer, ihre entschiedensten Gegner in Brunner und Kroenig fand. Auch die Methode der künstlichen Erzeugung steriler Abszesse im Körper (abcès de fixation) nach Fochier, die ebenfalls auf dem Gedanken einer Hyperleukocytose und Leukocytenablenkung aufgebaut ist, hat keine wertvollen Resultate gezeitigt.

Immer wieder begegnen wir in den therapeutischen Versuchen zur Bekämpfung der pyogenen Blutinfektion dem Gedanken, durch in die Blutbahn eingeführte Substanzen den Organismus von den pyogenen Erregern zu befreien, sei es im Sinne einer gedachten inneren Desinfektion durch Nutzbarmachung der bakterienabtötenden oder wachstumshemmenden Eigenschaften der eingeführten Substanz, oder durch eine beabsichtigte Kräftigung und Vermehrung der natürlichen Schutzvorrichtungen des Blutes selbst. Der Gedanke ist zu bestechend, die pyogene Infektion des Blutes in Parallele zu setzen mit der örtlichen eitrigen Entzündung, für welche uns das ganze Rüstzeug der heute gebräuchlichen Antiseptica zur Verfügung steht. Die schwerwiegende Frage ist aber die, wie werden diese antiseptischen Mittel in der Blutbahn und vom gesamten Organismus vertragen? Der Gedanke der ,inneren Desinfektion“ fand zuerst einen Ausdruck in der durch $\mathrm{Eh} \mathrm{rlich} \mathrm{eingeführten} \mathrm{Chemotherapie,} \mathrm{später}$ in der von Blumenthal vorgeschlagenen Verwendung des Silberatoxyls, in der Behandlung mit sehr verdünnten Sublimatlösungen ( $\mathrm{I}-5 \mathrm{ccm}$ einer Lösung $\mathrm{I}: \mathrm{rooo}$ ) nach Bacelli, mit Magnesium sulfuric. nach $\mathrm{Hugg}$ ins und in letzter Zeit in der von Morgen rot $h$ ins Leben gerufenen Therapie mit Chininderivaten (Äthylhydrocuprein-Vuzin). Alle oben angeführten Mittel haben in der Behandlung der Blutinfektion ihre Erfolge, aber auch ihre Mißerfolge gehabt. Es ist zu erwarten, daß dasselbe Schicksal noch weiteren Versuchen in dieser Richtung erwachsen wird, bis wir mit verbesserten bakteriologischen und serologischen. Untersuchungsmethoden immer mehr Kenntnis von den Lebensäußerungen und Lebensbedingungen der pyogenen $\mathrm{Er}$ - 
reger im Blut und dem weiteren Schicksal der von uns in die Blutbahn eingeführten Substanzen erhalten. Bis heute sind die von uns intravenös gegebenen Mittel vom Momente der Injektion $\mathrm{ab}$ unserer genauen Kenntnis und Einwirkung entzogen und nur Bruchteile von Abbauprodukten in den uns zugänglichen Sekreten des Körpers, oder einige bakteriologische und serologische Untersuchungen geben uns unvollkommenen AufschluB über das Schicksal der in die Blutbahn eingeführten artfremden Substanz. So bleibt uns immer noch die Notwendigkeit, den therapeutischen Wert oder Unwert eines Mittels in der Blutbahn zu messen und zu beurteilen nach dem offensichtlichen klinischen Erfolg oder Mißerfolg. Nach diesen Gesichtspunkten kann es nicht verwundern, daß bei dem schweren und prognostisch ernsten, dabei aber so häufigen Krankheitsbilde der pyogenen Blutinfektion der Arzt, durch die Aussichtslosigkeit jeder kausalen Therapie veranlaßt, zu fast jedem Mittel greift, das ihm als relativ unschädlich bekannt ist, und an dieses die Hoffnung knüpft, diese gefährliche Krankheit wirksam zu bekämpfen.

Nach den soeben aufgeführten Gesichtspunkten wurden an der hiesigen Klinik von mir therapeutische Versuche mit intravenösen Urotropin-Injektionen bei einigen Fällen von zum Teil recht schweren chirurgischen pyogenen Blutinfektionen gemacht mit teilweise so günstigem klinischen Erfolg, daß ich nach reiflicher Überlegung und Prüfung des Materials mich entschlossen habe, die Fälle zu beschreiben und zu einer Nachprüfung der Methode aufzufordern. Der Gedanke, das Urotropin in die Blutbahn einzuführen, ist in den letzten Jahren seit der Bekanntgabe des Mittels wiederholt aufgetaucht und auch von einigen Autoren in die Wirklichkeit übertragen worden. Deuts ch gab 1919 eine Behandlungsmethode des Gelenkrheumatismus mit intravenösen Urotropininjektionen an, $\mathrm{S}$ a $\mathrm{c}$ h s eine solche bei Erkrankung durch Trichophytoninfektion und später bei Angina necrotica, Angina lacunaris, sowie einigen Formen von Stomatitis; L oeper und Grosdidier behandelten die verschiedensten Infektionskrankheiten, unter andern auch einige Tuberkulosefälle, mit Urotropin intravenös. Trotz der zahlreichen Arbeiten, die in letzter Zeit über die orale, subkutane und intravenöse Anwendung des Urotropins erschienen sind, besteht noch keine vollständige Klarheit 
weder über die Wirkung des Mittels, noch über sein Schicksal in der Blutbahn. Das Urotropin (Hexamethylentetramin) wurde am Ende des 19. Jahrhunderts von $\mathrm{Nicolaier}$ in die Therapie eingeführt. Er nahm an, daB die bakterizide Wirkung desselben auf der Abspaltung von Formaldehyd beruhe. Diese Abspaltung sollte aber nur in saurer Lösung und bei Körperwärme stattfinden, wobei das Urotropin in Ammoniak und Formaldehyd sich zersetzen sollte. Von dem per os eingenommenen Urotropin erscheint ein großer Teil wieder unzersetzt im Urin, während ein anderer Teil des Präparats in den Nieren (?) das Formaldehyd abspaltet, das als solches dann im Urin nachweisbar ist. Zum Nachweis des letzteren im Urin sind eine Anzahl zum Teil recht scharfer chemischer Proben angegeben worden, vor allem die J or riss en sche Probe mit Phlorogluzin, dann andere Proben von Lebbin, Bayer-Tollens, Burmann und Arnold. Die empfindlichste Probe auf Formaldehyd scheint die von $S \mathrm{chr}$ der mit Phenylhydracin zu sein. Ich habe bei meinen Versuchen diese, sowie die von $G$ r o $B$ angegebene Sublimatprobe regelmäßig angestellt, und es gelang mir öfters, noch verschwindende Spuren von Formaldehyd $(0,0001 \mathrm{~g})$ im Urin nachzuweisen, bei den Kontrollen besonders gut in saurer Lösung. Auch bei der Einführung des Urotropins in die Blutbahn erscheinen im Urin sowohl unverändertes Urotropin als auch Formaldehyd. Eine gute und brauchbare Probe zum Nachweis des unveränderten Urotropins gibt $\mathrm{Nicolaie} \mathbf{r}$ an. Bei Zusatz von Bromwasser (Brom Io,o, Aqua dest. 90,0) bildet sich bei kleinsten Mengen gelösten Urotropins ein charakteristischer orangegelber Niederschlag, das Lösungsmittel muß frei von Eiweiß sein. $O b$ es sich bei dem Formaldehydbefund im Urin um vom Urotropin abgespaltenes For. maldehyd oder aber urn im Urotropin als solchem schon enthaltenem freien Formaldehyd handelt, läßt sich zurzeit noch nicht sicher entscheiden.

Bakteriologisches.

Aus den Versuchen von Hanzlik, der in formaldehydfreiem, urotropinhaltigem Agar, der mit Ammoniak versetzt war, eine deutliche Wachstumshemmung bei Bact. typhi, Bact. coli, dysenteriae usw. fand, geht mit Sicherheit hervor, daB die bak- 
terienabtötende Wirkung des Urotropins sowohl dem abgespaltenen Formaldehyd als auch dem Urotropin selbst zuzusprechen ist. Ohira hat diese Versuche nachgeprüf und spricht nach seinen Resultaten dem Urotropin selbst keine bakterizide Wirkung zu. Diese Verschiedenheit der Ergebnisse beider Untersucher habe ich versucht aufzuklären. Bei der Beurteilung der Versuche Ohiras scheint mir der alkalisierte, vorher mit Äther abgedampfte Urin als Kulturmedium nicht geeignet. Um den wirklichen Verhältnissen nahezukommen, ist es erforderlich, ein Nährmedium zu wählen, das den Körperverhältnissen möglichst entspricht. Am geeignetsten erschien mir daher als Nährsubstrat das reine menschliche Serum und die Serumbouillon, als Untersuchungstemperatur die Körperwärme ( $37 \mathrm{Grad})$. Ich habe zur Ermittlung der bakteriziden Wirkung des Urotropins in der Form, wie wir es in die Vene einführen, d. h. in 40proz. Lösung in physiologischer Kochsalzlösung, eine Reihe bakterjologischer Reagenzglasversuche gemacht und dabei die verschiedenen, als pyogene Erreger in der Blutbahn bekannten Bakterien, mit dem Urotropin zusammengebracht. Als Bakterienmaterial wurden nur 24stündige Agarkulturen aus frischem Eiter verwandt. Die Wachstumshemmung oder Abtötung wurde derart geprüft, daß nach 24stündigem Verweilen der Kulturen im Brutschrank bei 37 Grad mit Urotropinzusatz je $3 \mathrm{~N}$-Ösen der Kulturflüssigkeit auf frischen Nährboden übertragen wurden (Schrägagar), und erst von diesen Agarkulturen das Endresultat nach abermaligem 24 stündigem Verweilen im Brutschrank abgelesen wurde. Die Ergebnisse dieser Versuche habe ich in Tabelle I zusammengestellt. Es ergab sich, $\mathrm{da} B$ die bakterizide Kraft des Urotropins gegenüber den einzelnen Bakterienarten verschieden ist. Diese Erscheinung ist nicht absonderlich, wenn man die Versuche mit ähnlichen Prüfungen anderer Antiseptica in Parallele setzt. Eine starke bakterizide Wirkung, verglichen mit den bekannten antiseptischen Mitteln, vor allem den modernen Chininderivaten, kann dem Urotropin nicht zugesprochen werden. Ausgesprochene Wachstumshemmung oder Abtötung treten erst bei relativ hohem Prozentgehalt der Urotropinkonzentration ein. Die stärkste Wirkung entfaltet es gegen Pneumokokken, Streptococc. longus und Bact. pyocyaneus, weniger gegen Staphylokokken und die geringste gegen Bact. coli 
Tabelle I.

\begin{tabular}{|c|c|c|c|c|}
\hline $\begin{array}{c}\text { Röhrchen } \\
\mathrm{Nr} .\end{array}$ & Nährboden & Impfmaterial & $\begin{array}{l}\text { Urotropin- } \\
\text { zusatz }\end{array}$ & Ergebnis nach 24 Stunden \\
\hline I & 5 ccm Nähr- & - & 一 & Kein Wachstum \\
\hline II & sgl. & $\begin{array}{l}\text { I N-Öse Sta- } \\
\text { phylococcus }\end{array}$ & - & Starkes Wachstum \\
\hline III & desgl. & $\begin{array}{l}\text { pyog. aur. } \\
\text { desgl. }\end{array}$ & $1 \%$ & $\begin{array}{c}\text { Starkes Wachstum; keine } \\
\text { Hemmung }\end{array}$ \\
\hline IV & desgl. & desgl. & $2 \%$ & Ausgespr. Wachstumshemmung \\
\hline $\mathrm{V}$ & desgl. & desgl. & $2,5 \%$ & Abtötung \\
\hline$\sqrt{1}$ & des & desgl. & & AL \\
\hline I & $\begin{array}{l}5 \mathrm{ccm} N a ̈ h r- \\
\text { bouillon }\end{array}$ & - & - & Kein Wachstum \\
\hline II & desgl. & $\begin{array}{l}3 \text { N.-Ösen } \\
\text { Pneumococ. }\end{array}$ & - & Sehr starkes Wachstum \\
\hline III & desgl. & desgl. & $0,6 \%$ & Ausgespr. Wachstumshemmung \\
\hline IV & desgl. & desgl. & $1,2 \%$ & \\
\hline $\begin{array}{l}\mathrm{V} \\
\mathrm{VI}\end{array}$ & desgl. & desgl. & $1,8 \%$ & Abtötung \\
\hline VII & desgl. & desgl. & $\begin{array}{l}2,4 \% \\
3 \%\end{array}$ & ADtutung \\
\hline VIII & desgl. & desgl. & $6 \%$ & \\
\hline $\mathrm{N}$ & & & & \\
\hline I & $10 \mathrm{ccm}$ & - & - & Kein Wachstum \\
\hline II & $\begin{array}{l}\text { Blutagar } \\
\text { desgl. }\end{array}$ & $\begin{array}{l}\text { I N.Öse Strep- } \\
\text { tococcus long. }\end{array}$ & - & $\begin{array}{c}\text { Nach } 48 \text { Stunden } \\
\text { Sehr starkes Wachstum }\end{array}$ \\
\hline III & desgl. & desgl. & $0,6 \%$ & Deutliche Wachstumshemmung \\
\hline IV & desgl. & desgl. & $1,0 \%$ & Starke Wachstumshemmung \\
\hline V & desgl. & desgl. & $1,5 \%$ & Abtötung \\
\hline $\begin{array}{l}\text { Röhrchen } \\
\text { Nr. }\end{array}$ & & & & \\
\hline I & $\begin{array}{l}5 \mathrm{ccm} N a ̈ h r \\
\text { bouillon }\end{array}$ & - & - & Kein Wachstum \\
\hline II & desgl. & $\begin{array}{l}\text { I N.Öse Bact. } \\
\text { coli com. }\end{array}$ & - & Sehr starkes Wachstum \\
\hline III & desgl. & desgl. & $1 \%$ & Geringeres Wachstum \\
\hline V & $\begin{array}{l}\text { desgl. } \\
\text { desgl. }\end{array}$ & $\begin{array}{l}\text { desgl. } \\
\text { desgl. }\end{array}$ & $\begin{array}{l}2 \% \\
3 \%\end{array}$ & $\begin{array}{c}\text { Ausgesprochene Wachstums- } \\
\text { hemmung }\end{array}$ \\
\hline $\begin{array}{l}\text { VI } \\
\text { VII }\end{array}$ & $\begin{array}{l}\text { desgl. } \\
\text { desgl. }\end{array}$ & $\begin{array}{l}\text { desgl. } \\
\text { desgl. }\end{array}$ & $4 \%$ & Starke Wachstumshemmung \\
\hline VIII & $\begin{array}{l}\text { desgl. } \\
\text { desgl. }\end{array}$ & $\begin{array}{l}\text { desgl. } \\
\text { desgl. }\end{array}$ & $\begin{array}{l}3 \% \\
6 \% \\
7 \%\end{array}$ & Abtötung \\
\hline I & $5 \mathrm{ccm} N a ̈ h r-$ & - & - & Kein Wachstum \\
\hline II & $\mathrm{de}$ & $\begin{array}{l}\text { I N-Öse Baz. } \\
\text { pyocyaneus }\end{array}$ & - & Sehr starkes Wachstum \\
\hline $\begin{array}{l}\text { III } \\
\text { IV }\end{array}$ & $\begin{array}{l}\text { desgl. } \\
\text { desgl. }\end{array}$ & $\begin{array}{l}\text { desgl. } \\
\text { desgl. }\end{array}$ & $\begin{array}{l}1 \% \\
1,5 \%\end{array}$ & Ausgespr. Wachstumshemmung \\
\hline$\underset{V T}{V}$ & & desgl. & $2 \%$ & Abtötung \\
\hline VII & desgl. & desgl. & - $2,9 \%$ & \\
\hline
\end{tabular}




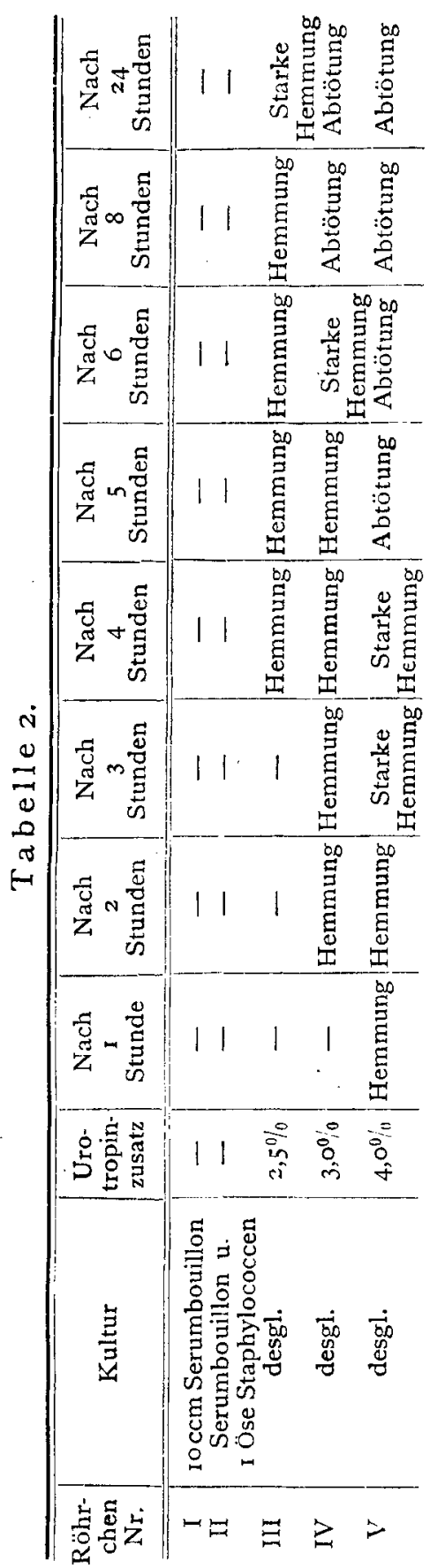

comm. Ich kann somit die Resultate von Deutsch im großen und ganzen bestätigen, wenn ich auch die Methode seiner Untersuchungen für nicht völlig ausreichend halte, um Wachstumshemmung oder Abtötung von Bakterien einwandfrei festzustellen. Um den Zeitpunkt des Einsetzens der bakteriziden Wirkung festzustellen, machte ich eine andere Reihe von Versuchen in menschlicher Serumbouillon, um den Körperverhältnissen möglichst nahezukommen. Die Ergebnisse dieser Versuchsreihe sind aus Tabelle 2 ersichtlich. Es ergab sich dabei, daß eine volle bakterienabtötende Wirkung erst nach 5 Stunden zur Geltung kommt, während Wachstumshemmung schon erheblich früher einsetzt. Die Resultate wurden wieder gewonnen durch stundenweises Übertragen der Kulturflüssigkeit zu 3 N-Ösen auf frischen Nähragar, um eine weitere Berührung mit dem Urotropin vollständig auszuschalten.

Zur Orientierung, ob die gewonnenen Resultate nur dem Formaldehyd oder vieleicht sogar nur dem Lösungsmittel (Kochsalzlösung) zuzusprechen sind, wurden andere Versuche angestellt, in denen ich diese beiden Komponenten isoliert in gleicher Versuchsanordnung zum Bakterienmaterial und der Kulturflüssigkeit zusetzte. Die Resultate zeigt Tabelle 3. Es ist aus diesen 
Tabelle 3 .

\begin{tabular}{|c|c|c|c|c|}
\hline $\begin{array}{c}\text { Röhrchen } \\
\text { Nr. }\end{array}$ & Nährboden & Impfmaterial & Zusätze & $\begin{array}{l}\text { Ergebnis nach } \\
24 \text { Stunden }\end{array}$ \\
\hline 1 & $5 \mathrm{ccm}$ Nährbouillon & $\begin{array}{c}\text { I N.-Öse } \\
\text { Staphyl. pyog. aureus }\end{array}$ & - & \\
\hline $\begin{array}{l}\text { II } \\
\text { III } \\
\text { IV } \\
V \\
\text { VI }\end{array}$ & $\begin{array}{l}\text { desgl. } \\
\text { desgl. } \\
\text { desgl. } \\
\text { desgl. } \\
\text { desgl. }\end{array}$ & $\begin{array}{l}\text { desgl. } \\
\text { desgl. } \\
\text { desgl. } \\
\text { desgl. } \\
\text { desgl. }\end{array}$ & $\begin{array}{l}\mathrm{NaCl} \\
0,6 \% \\
1,2 \% \\
1,8 \% \\
2,4 \% \\
3,0 \%\end{array}$ & $\begin{array}{c}\text { Starkes } \\
\text { ungehindertes } \\
\text { Wachstum }\end{array}$ \\
\hline I & $5 \mathrm{ccm}$ Nährbouillon & $\begin{array}{c}\text { I N.-Öse } \\
\text { Staphyl. pyog. aureus }\end{array}$ & $\begin{array}{l}\text { Formal. } \\
\text { deh d } \\
0,6 \%\end{array}$ & \\
\hline $\begin{array}{l}\text { II } \\
\text { III } \\
\text { IV } \\
\text { V }\end{array}$ & $\begin{array}{l}\text { desgl. } \\
\text { desgl. } \\
\text { desgl. } \\
\text { desgl. }\end{array}$ & $\begin{array}{l}\text { desgl. } \\
\text { desgl. } \\
\text { desgl. } \\
\text { desgl. }\end{array}$ & $\begin{array}{l}1,2 \% \\
1,8 \% \\
2,4 \% \\
3,0 \%\end{array}$ & Kein Wachstum \\
\hline
\end{tabular}

Tabelle 4 .

\begin{tabular}{|c|c|c|c|c|}
\hline $\begin{array}{l}\text { Röhr- } \\
\text { chen } \\
\text { Nr. }\end{array}$ & Nährboden & Impfmaterial & $\begin{array}{c}\text { Uro- } \\
\text { tropin- } \\
\text { zusatz }\end{array}$ & $\begin{array}{l}\text { Ergebnis nach } \\
24 \text { Stunden }\end{array}$ \\
\hline $\begin{array}{l}\text { II } \\
\text { III } \\
\text { IV } \\
V \\
\text { VI }\end{array}$ & \begin{tabular}{|l}
$5 \mathrm{ccm}$ Nährbouillon \\
u. I Tr. Normal HCl \\
desgl. \\
desgl. \\
desgl. \\
desgl. \\
desgl.
\end{tabular} & \begin{tabular}{|c|}
$3 \mathrm{~N}$. -Ösen \\
Staphyloc. pyog. aureus \\
desgl. \\
desgl. \\
desgl. \\
desgl. \\
desgl.
\end{tabular} & $\begin{array}{l}- \\
0,6 \% \\
1,2 \% \\
1,8 \% \\
2,4 \% \\
3 \% \\
\% \\
0 \%\end{array}$ & $\begin{array}{l}\text { Starkes Wachstum } \\
\text { Wachstumshemmung } \\
\left\{\begin{array}{l}\text { Starke Wachs- } \\
\text { tumshemmung } \\
\text { Abtötung }\end{array}\right.\end{array}$ \\
\hline I & $\begin{array}{l}5 \mathrm{ccm} \text { Nährbouillon } \\
\text { u. I Tr. Normal } \mathrm{NaOH}\end{array}$ & $\begin{array}{c}3 \text { N.-Ösen } \\
\text { Staphyloc pyog. aureus }\end{array}$ & - & Starkes Wachstum \\
\hline $\begin{array}{r}\text { II } \\
\text { III } \\
\text { IV } \\
\text { V } \\
\text { VI }\end{array}$ & $\begin{array}{l}\text { desgl. } \\
\text { desgl. } \\
\text { desgl. } \\
\text { desgl. } \\
\text { desgl. }\end{array}$ & $\begin{array}{l}\text { desgl. } \\
\text { desgl. } \\
\text { desgl. } \\
\text { desgl. } \\
\text { desgl. }\end{array}$ & $\begin{array}{l}0,6 \% \\
1,2 \% \\
1,8 \% \\
2,4 \% \\
3,0 \%\end{array}$ & $\begin{array}{l}\text { Wachstumshemmung } \\
\left\{\begin{array}{l}\text { Starke Wachs- } \\
\text { tumshemmung } \\
\text { Abtötung }\end{array}\right.\end{array}$ \\
\hline
\end{tabular}

Versuchen ersichtlich, daß dem Kochsalz in diesen Konzentrationen überhaupt keine bakterizide Wirkung (entgegen Ohira), dem reinen Formaldehyd aber eine erheblich stärkere als dem Urotropin zukommt. Ebenfalls wurden in derselben Anordnung Versuche gemacht, um zu prüfen, ob ein vermehrter Säuregehalt oder eine vermehrte Alkaleszenz des Nährmediums eine entscheidende Einwirkung auf die bakterizide Wirkung des Urotropins ausübt (s. Tabelle 4). Ich konnte dabei eine Verschiedenheit der 
Wirkung in saurem oder alkalischem Nährmittel bei 37 Grad nicht feststellen, höchstens daß die Wachstumshemmung im sauren Nährboden ausgesprochener war; eine Abtötung trat nicht früher ein.

Es hat sich also aus den bakteriologischen Reagenzglasversuchen ergeben, daß dem Urotropin als solchem bei Körpertemperatur im menschlichen Serum keine erhebliche bakterizide Wirkung zuzusprechen ist, und daß sowohl Wachstumshemmung wie Abtötung erst nach mehrstündigem Verweilen des Urotropins im Kulturmedium auftritt. Es erhebt sich nun die Frage: Wie lassen sich die klinischen Erfahrungen, vor allem die Resultate der bakteriologischen Blutuntersuchung am Krankenbette nach intravenösen Urotropininjektionen mit der verhältnismäßig geringen Wirkung im Reagenzglase in Einklang bringen? Aus den Reagenzglaśversuchẹ mußte man den Schluß ziehen, daß eine sehr große Menge Urotropin in die Blutbahn eingeführt werden muß, um einen therapeutischen Erfolg zu garantieren. Bei der rein theoretischen Annahme der durchschnittlichen Blutmenge des erwachsenen Menschen von $2000 \mathrm{ccm}$ wären nach den Versuchen I $5 \mathrm{~g}$ Urotropin erforderlich, die auf einmal in die Blutbahn eingeführt, eine sofortige Abtötung sämtlicher darin enthaltenen pyogenen Keime erreichen könnten. Die klinische Erfahrung, gestützt auf bakteriologische Blutuntersuchungen, hat aber gelehrt, daß schon erheblich geringere Mengen des Mittels in der Blutbahn eine deutliche, wenn auch nicht abgeschlossene therapeutische Wirkung im Körper hervorbringen. Der therapeutische Erfolg geringer Mengen läßt sich in Wirklichkeit in exakter und eindeutiger Weise durch das kulturelle Züchtungsverfahren der pyogenen Erreger aus dem Blut nachweisen." Tatsächlich scheinen die Verhältnisse im Organismus von denen in vitro grundverschieden zu sein. Auch der Tierversuch ist aus oben näher angegebenen Gründen nicht zum Beweise von Vorgängen im kranken und geschädigten menschlichen Körper heranzuziehen. Selbst der Nachweis des Formaldehyds oder Urotropins im Blut oder Serum genügen in dieser Hinsicht nicht. Wir wissen fast gar nichts über die Ablagerung oder die Verteilung der in das Blut eingeführten Substanz im Körper, noch weniger über die Wirkung im Blut. Auch die quantitative Messung der 
ausgeschiedenen Substanz in den uns zugänglichen Körpersekreten gibt uns nur unvollkommenes Beweismaterial für das Vorhandensein im Körper, nicht für deren Wirkung. Zu bedenken ist ferner, daß die große Menge des Bakterienmaterials, wie wir sie im Reagenzglasversuch zur sicheren Demonstration der Verhältnisse verwenden müssen, kaum zu vergleichen sind mit den relativ verschwindend wenig Keimen in der Blutbahn. Andererseits sind im Organismus Kräfte wirksam, zum Teil abwehrende, zum Teil fördernde (Phagocytose, Leukocytose, Abwehrfermente, Virulenz), die wir niemals im Reagenzglase nachahmen können. Man könnte aus den Versuchen in vitro folgern, daß die bakterizide Wirkung des Urotropins in der Blutbahn in zwei Komponenten zerfällt, eine dem Urotropin gehörige, bestehend in der langsamen Schädigung des Nährbodens durch Abspaltung von Ammoniak, die andere dem abgespaltenen Formaldehyd gehörige in der schließlichen Vernichtung der schon geschädigten Erreger durch die stärkere bakterizide Kraft des Formaldehyds. Jedoch ist auch dieses eine Hypothese, für die wir zurzeit noch keine vollbeweisenden Untersuchungsmethoden besitzen.

$\mathrm{Ob}$ durch das Einführen von Urotropin in die Blutbahn das Blut selbst, also der Nährboden für die Bakterien, verändert wird, ist fraglich. Vielleicht werden aber im Blute Substanzen abgelagert oder verweilen jedenfalls längere Zeit nach Urotropininjektionen in der Blutbahn, die nicht nur im menschlichen Organismus, sondern auch im Reagenzglasversuch bakterienabtöten'd oder wachstumshemmend wirken. Dieses konnte ich durch eine Versuchsanordnung nachweisen, die mir auch sonst geeignet erscheint, über die Wirkung in die Blutbahn eingeführter Substanzen Aufschluß zu verschaffen.

Einem gesunden Menschen wurden aus der Armvene $5 \mathrm{ccm}$ Blut entnommen, dasselbe auf 5 Agarröhrchen zu je Io ccm Agar verteilt und jedes Röhrchen dann mit gleicher Menge Bakterienmaterial beimpft. Verwandt wurde eine Kochsalzaufschwemmung von Staphylokokken aus frischen 24 stündigen Agarkulturen, von der zu jedem Röhrchen je $0,5 \mathrm{ccm}$ zugesetzt wurden. Nachdem Blut und Bakterienmaterial in den Agarröhrchen gut verteilt waren, wurde jedes Röhrchen in ein steriles Petrischälchen ausgegossen und die fertigen Blutplatten auf 24 Std. in den Brutschrank gestelit. Dieser erste Versuch diente als Kontrolle. Nach 24 Std. ließ sich durch Auszählen 
der Kolonien auf jeder Blutplatte das Wachstum feststellen. Dann erhielt die Versuchsperson 8,og Urotropin in der von uns angewandten Lösung (40 proz., in steriler, physiolog. Kochsalzlösung) intravenös. Es wurden nun nach $1 / 4$ Std., nach 2 , nach 8 und 12, dann nach 24 Std. je $5 \mathrm{ccm}$ Blut aus einer Vene des anderen, nicht injizierten Armes entnommen und jedesmal mit gleicher Menge Bakterienmaterial und flüssigem Agar zu 5 Blutplatten verarbeitet. Alle Blutplatten wurden zuerst nach 24 Std., endgültig nach 48 Std. abgelesen. Zusammenfassend hatten die Versuche bei intravenösen Urotropingaben von $8,0 \mathrm{~g}$ folgendes Resultat:

\begin{tabular}{|c|c|c|c|c|c|c|c|c|}
\hline & \multicolumn{6}{|c|}{ 1. Vor Urotropin im ccm Blut 308 Keim } \\
\hline 3.2 & " & " & " & " & " & , & 5 & , \\
\hline 4. 8 & " & $"$ & " & $"$ & " & " & - & \\
\hline I 2 & , & " & , & " & , & $"$ & I & \\
\hline 24 & , & , & $"$ & " & , & , & I 10 & \\
\hline
\end{tabular}

Danach ist die stärkste Wirkung nach 8-I2 Std. zu erwarten, während nach 24 Std. die Wirksamkeit des Urotropins wieder aufgehoben ist.

Ich glaube, daß man berechtigt ist, bei einer so schweren und prognostisch ernsten Krankheit wie der pyogenen Blutinfektion ein Mittel anzuwenden, das nach zahlreichen therapeutischen Versuchen in angemessener Dosierung vom Kranken ohne Schädigung vertragen wird, wenn auch seine bakterizide Kraft im Reagenzglase weit zurückbleibt hinter anderen, dafür aber gefährlicheren, antiseptischen Substanzen.

Klin is ches.

Seit Oktober 1920 habe ich an der hiesigen Klinik bei dem immerhin nicht geringen Material an chirurgischen pyogenen Blutinfektionen das Urotropin intravenös gegeben. Ich möchte an dieser Stelle sogleich betonen, daß ich zu den therapeutischen Versuchen angeregt wurde durch eine Notiz, die ich in der Literatur fand. In einer Sitzung der medizinischen Sektion der Schlesischen Gesellschaft für vaterländische Kultur zu Breslau stellte Alker einen Fall von pyogener Blutinfektion durch Staphylokokken vor, den er mit intravenösen Urotropininjektionen erfolgreich behandelt hatte. Ich hatte damals gerade eine sehr schwere Staphylomykose in Behandlung, bei der wiederholte Fulmargininjektionen keinen Erfolg gebracht hatten (Fall I). Ich wandte dann das Urotropin intravenös an und war überrascht durch den 
guten Erfolg. Darauf beschloß ich, das Mittel weiter bei der Behandlung der pyogenen Blutinfektion zu versuchen, und hatte im Verlauf von 6 Monaten Gelegenheit, I7 weitere Fälle, zum Teil recht schwere Infektionen, damit $\mathrm{zu}$ behandeln. Bei der vorher erwähnten Demonstration eines Falles von $A l k e r$ fand sich keinerlei Angabe über Menge und Dosierung des zu verwendenden Urotropins. Ich entschloß mich bald, geleitet durch die vorher angestellten Reagenzglasversuche und die vorgefundenen Berichte in der sonstigen Urotropinliteratur, eine möglichst hochkonzentrierte Lösung zu verwenden, um möglichst viel Substanz in möglichst wenig Lösungsmittel in die Blutbahn zu bringen. Verwandt wurde nur eine 4oproz. (höchstprozentige) Lösung, von der die Hersteller (Chem. Fabr. a. Akt. vorm. E. Schering, Berlin, N. 39) betonen, daB sie in der Kälte ihre Urotropinkonzentration nicht einbüßt und ihren Urotropingehalt Monate hindurch unzersetzt behält. Als Lösungsmittel verwandte ich nach anfänglichen Versuchen mit Aqua dest. später nur physiologische Kochsalzlösung, weil ich bestimmt glaube, daß unangenehme Nebenerscheinungen, wie von einzelnen Autoren beschrieben und auch von mir an den ersten Fällen beobachtet (Übelkeit, Temperaturanstieg, örtliche Gewebsnekrosen an der Einstichstelle) auf das Aqua dest. als Lösungsmittel zurückzuführen sind. Die Lösung wurde jedesmal vor dem Gebrauch durch ro Minuten langes Aufkochen im Wasserbade erneut sterilisiert. Auch ist es ratsam, die Lösung vor der Injcktion auf Körperwärme zu bringen, da sich hierdurch ein unangenehmes Brennen an der Injektionsstelle, das etwa 3-5 Minuten dauert und dadurch hervorgerufen wird, daß einige Tropfen der Injektionsflüssigkeit in das perivaskuläre $\mathrm{Ge}$ webe gelangen, am sichersten vermeiden läßt. Ferner kann diese momentane Unannehmlichkeit der Injektion noch mehr vermieden werden durch vorheriges sorgfältiges Abwischen der Injektionskanüle und Reinigung derselben von jeder Spur anhaftenden Urotropinj. Bei derartigem Verfahren wurde später bei keinem Patienten mehr, trotz täglicher Injektionen, Brennen an der Injektionsstelle beobachtet. Nach der Injektion haben die Patienten keinerlei charakteristische Erscheinungen mit Ausnahme eines ziemlich starken Schweißausbruchs nach I-2 Stunden, der zugleich auch das Absinken der Temperatur ankündigt. Örtliche 
Gewebsnekrosen an der Injektionsstelle auch bei wiederholtem absichtslosem Injizieren einiger Tropfen in das subkutane Gewebe habe ich niemals gesehen.

Eine genaue Angabe über die Größe der Einzeldosis läßt sich bei der geringen Zahl der behandelten Fälle noch nicht geben. Genügend zur therapeutischen Wirksamkeit und ausreichend zur baldigen Unterdrückung der blutinfektiösen Symptome waren tägliche Einzeldosen von $4-6 \mathrm{~g}$ in einmaliger Injektion ( $10-15 \mathrm{ccm}$ einer 4oproz. Lösung). Höhere Einzeldosen oder mehrmalige tägliche Darreichung derselben Dosis halte ich nicht für günstiger, trotzdem sie ebenso schadlos vertragen werden. Durch zu hohe Einzeldosen wird nämlich das Ende der Therapie mit Urotropin unliebsam beschleunigt. Die Behandlung muß sofort ausgesetzt werden, sobald eine charakteristische Blasenreizung eintritt. Bezüglich dieser einzigen Komplikation, die in manchen (nicht allen) Fällen nach etwa 6-8 Injektionen auftritt und von manchen Autoren als Nierenschädigung angesprochen wird, möchte ich bemerken, daß es sich dabei um eine Entzündung der Blasenschleimhaut handelt, die vielleicht auf das längere Verweilen von formaldehydhaltigem Urin in der Blase zurückzuführen ist. In einem der von mir behandelten Fälle, der wegen der Schwere des Krankheitsbildes an mehreren Tagen hintereinander mit einmaligen starken Urotropininjektionen (8 $\mathrm{g}$ täglich) behandelt war, traten diese Blasenerscheinungen am 7. Behandlungstage in Gestalt von leichtem Brennen beim Wasserlassen, Hämaturie und vermehrtem Harndrang auf. Die Beschwerden gingen im Laufe eines Tages vollkommen zurück, es blieb aber eine leichte Hämaturie und Albuminurie. Der Patient kam am II. Behandlungstage infolge eines seit 40 Jahren bestehenden schweren Herzfehlers ad exitum und gelangte zur Obduktion (Fall I7). Bei genauer Untersuchung der Organe von pathologischer Seite fanden sich in beiden Nieren keinerlei akute entzündliche Veränderungen, die auf die vorher durchgemachte Urotropinbehandlung zurückzuführen gewesen wären, dagegen zeigte die Blasenschleimhaut zahlreiche punktförmige frische Kapillarblutungen, keine eitrigen Auflagerungen, im ganzen einen akuten Entzündungszustand, der fraglos seine Ursache in der Urotropinbehandlung hatte. Für gewöhnlich gehen diese Blasenerscheinungen nach Aussetzen 
der Urotropinbehandlung in kurzer Zeit, spätestens in 24 Stunden, vollständig zurück. Jedoch geben sie immer das Signal zum sofortigen Aufhören mit der Urotropintherapie. Diese Komplikation ist auch von anderen Autoren wiederholt beobachtet und von einigen auch in gleicher Weise gedeutet worden ( $\mathrm{Ni}$ col a ier, Deutsch, GroB, Sachs). Es wird sich daher die Dauer der intravenösen Anwendung des Urotropins bestimmen lassen aus dem klinischen Erfolg (Abfall der Temperatur, Aufhören der Schüttelfröste, Verschwinden der Bakterien aus den Blutkulturen); andererseits durch 'das Auftreten der vorher beschriebenen Blasenentzündung. Andere Komplikationen habe ich nicht gesehen, sind meines Wissens auch nicht in der Literatur beschrieben worden. $\mathrm{Ob}$ die längere intravenöse Anwendung des Urotropins mit der Zeit auch zu Nierenschädigungen führt, kann ich nicht sicher entscheiden. Ich habe in dem Urin der damit behandelten Patienten während der Behandlung und lange nach Abschluß derselben niemals Formelemente, die aus den Nieren stammten, nachweisen können.

Im allgemeinen wird die Behandlung einer mittelschweren pyogenen Blutinfektion bestehen in der täglichen intravenösen Gabe von 4-6 g Urotropin. Ich habe gefunden, daß danach die schweren Symptome aus dem Krankheitsbilde schon nach 2-3 Injektionen verschwunden sind. Die Bakterien sind nach 3-4 Injektionen aus der Błutbahn verschwunden. Die Blasenentzündung tritt meistens erst nach $6-8$ Injektionen auf. Im übrigen tritt die Blasenkomplikation nur in etwa I I Proz. der Fälle auf, nach anderen Autoren noch seltener ( $\mathrm{S} \mathrm{a} \mathrm{chs).} \mathrm{Es} \mathrm{ist} \mathrm{möglich} \mathrm{und} \mathrm{sogar}$ sehr wahrscheinlich, daß weit höhere Einzeldosen vom Organismus anstandslos vertragen werden, und besonders sehr schwere, hoffnungslose pyogene Blutinfektionen verführen wohl oft zu höheren Gaben. Jedoch setzt die vorher erwähnte Blasenentzündung dann oft der weiteren therapeutischen Anwendung des Urotropins ein unerwünscht frühes Ziel, weshalb in der Praxis von der Verwendung höherer Einzeldosen als $8 \mathrm{~g}$ täglich abzuraten ist.

Kontrolliert und gemessen wurde der therapeutische Erfolg des Mittels in der Blutbahn in jedem einzelnen Falle durch systematische kulturelle Blutuntersuchung. Gewöhnlich wurden sofort beim Beginn der Krankheitssymptome, nach operativen Eingrif- 
fen, nach mehreren Urotropininjektionen und dann innerhalb einiger Wochen vom Blute des Patienten Kulturen angelegt. Entnommen wurde das Blut in der allgemein gebräuchlichen Weise unter möglichst aseptischen Kautelen aus einer Cubitalvene. Entweder wurde das Blut mit steriler Injektionsspritze angesogen, oder es wurde durch die in die Vene eingestochene Kanüle in den vorher sterilisierten und im Wasserbade auf die notwendige Temperatur gebrachten Nährboden eingelassen. Die Methode des Ansaugens mit der Spritze bietet viele Vorteile, sie ist sauberer, dem Patienten angenehmer und schneller. Auch gestattet diese Methode nachträglich ein besseres Verteilen des Blutes auf das Nährmedium nach Tropfenzahl. Andererseits ist jeder Rückstand von Desinfizientien im Innern der Spritze sowie das notwendige Schütteln der Blutflüssigkeit in der Spritze vor der Verimpfung eine Fehlerquelle für genaue bakteriologische Resultate. Das Blut wurde sofort nach Entnahme in den bereitgehaltenen Nährboden übertragen. Ich entnahm stets $5 \mathrm{ccm}$ und verteilte diese Menge sogleich zu einem oder zwei Kubikzentimetern in flüssigem Nähragar, der dann in sterilen Petrischalen ausgegossen wurde. Die fertigen Platten kamen nach völligem Erkalten in den Brutschrank und wurden zum ersten Male nach 24 Stunden, endgültig nach 48 Stunden abgelesen. Das Plattenverfahren bietet gegenüber den Kulturen in flüssigem Nährboden (Zuckerbouillon mit Paraffinverschluß), sowie denen auf Schrägagar den großen Vorteil der besseren Übersichtlichkeit und Sichtbarmachung auch der kleinsten Bakterienkolonie. Geringes Bakterienwachstum auf den Platten wurde direkt nach Kolonien gezählt, bei stärkerem Wachstum wurde genaue Auszählung mit der Zählkammer vorgenommen. Nach dem Ansteigen oder der Abnahme der Bakterien in den Blutkulturen wurde der therapeutische Erfolg der Urotropininjektionen bewertet. Bei jeder Blutentnahme wurden mindestens 3 Platten gegossen. Die Zahlen in den Krankengeschichten bedeuten das Mittel der Kolonienanzahl aus sämtlichen Kulturen jeder Untersuchung.

$\mathrm{Zu}$ erwähnen ist noch, daß neben der spezifischen Therapie mit Urotropininjektionen die Allgemeinbehandlung unserer $\mathrm{Pa}$ tienten nicht zu kurz kam. Keine kausale Therapie der pyogenen Blutinfektion wird gute Erfolge zeitigen, wenn die auf Stärkung 
und Kräftigung des ganzen Organismus berechnete Allgemeinbehandlung in den Hintergrund gestellt wird. Zweckmäßige Ernährung, Sorge für häufige Darmentleerung, Körperpflege, Atemgymnastik, Stärkung der Herzkraft und andere Maßnahmen müssen jede Therapie der Blutinfektion wirksam unterstützen. Reichliche Flüssigkeitszufuhr per os und rektal als Kochsalztröpfcheneinlauf sind sehr wertvoll. Entschieden möchte ich auch bei der Behandlung der pyogenen Blutinfektion zur Darreichung nicht zu großer Mengen Alkohols in Gestalt von Wein oder verdünntem Kognak raten. Ich habe den Eindruck, daß tägliche geringe Mengen Alkohols, eine Flasche Wein oder $1 / 4$ J Kognak, den Zustand der Patienten guinstig beeinflussen.

Im folgenden gebe ich kurze Auszüge aus den Krankengeschichten der in der hiesigen Klinik seit Oktober 1920 mit intravenösen Urotropininjektionen behandelten pyogenen Blutinfektionen.

Krankengeschichten.

Fall I. K. D., 22 J. Schwere Kniegelenksvereiterung links nach alter Granatsplitterverletzung.

22. IX. I920. Operation: Eröffnung des Kniegelenks. Am I0. Krankheitstage bei sehr starker Eiterung aus den zahlreichen Inzisionswunden Temperaturaufstieg auf 39,8 Grad, Schüttelfrost, Übelkeit.

6. X. Die schweren Allgemeinstörungen, die den Verdacht einer Blutinfektion erweckten, haben täglich zugenommen, täglich 2 schwere Schüttelfröste, sehr häufiges Erbrechen, starke Eiterung. Am selben Tage in der Blutkultur sehr reichlich Staphylokokken, keine hämolytischen Streptokokken. Zunächst wird ein Versuch mit Fulmargin gemacht, ohne daß eine Beeinflussung der Temperatur oder der Blutinfektion zu verzeichnen war.

8. X. Urotropin $4, \mathrm{og}$ intravenös, ebenso die nächsten 3 Tage je eine Injektion von $6, \mathrm{og}$ intravenös, das gut vertragen wurde. I3. X. Temp. zur Norm abgefallen, keine Schüttelfröste, kein Erbrechen, völliges Wohlbefinden. Eiterung aus den Wunden noch ziemlich stark. Auf Blutplatten nur ganz vereinzelte Staphylokokken-Kolonien. 26. X. Pat. völlig geheilt entlassen. Wunden ganz verheilt. Seitdem keine Zeichen einer Blutinfektion.

F a ll 2. H. K., 10 J. Sehr schwere Osteomyelitis tibiae sin. acuta. Beginn der Erkrankung vor 5 Tagen nach einem Sturz auf dem Eise. Das schr elende, hochfiebernde Kind wird in schwer krankem Zuistand eingeliefert, mehrere Schüttelfröste täglich. In sofortigen Blutkulturen massenhaft Streptococc. longus haemolyt. In beiden 
Lungen schwere Veränderungen, die als metastatische Herde angesprochen wurden.

3. XII. Operation: Osteotomia tibiae.

5. XII. Verschlechterung des Allgemeinbefindens, hohe Temp., starke Atemnot, starke Durchfälle. Im Blut massenhaft Bakterien.

6. XII. 3,og Utropin i. v. ebenso in den nächsten 5 Tagen. 12. XII. Schüttelfröste haben nachgelassen, ebenso die Durchfälle; Temp. immer noch sehr hoch. Starke Atembeschwerden. Herzkraft sehr schlecht. Zeitweise benommen. I8. XII. Exitus letalis unter Zeichen schwerster Atemnot und Herzschwäche.

Obduktion: Umfangreiche metastatische Herde in beiden Lungen. Schwere Osteomyelitis tibiae sin. Keine Metastasen in den Nieren. Metastatische Ulzerationen im Rektum.

Fall 3. L. W., I3 J. Osteomyelitis tibiae sin. acuta.

Vor 6 Wochen auf das lk. Knie gefallen. Beginn der jetzigen Krankheit vor 4 Tagen mit heftigen Schmerzen im lk. Kniegelenk und $\mathrm{lk}$. Unterschenkel. Hohe Temperaturen und Schüttelfröste, kein Erbrechen. Im Blut sehr wenig Kolonien von Staphylococc. pyog. aur.

I. XII. Operation: Osteotomia tibiae sin. Starker eitriger Zerfall des Knochenmarks und parartikuläre Phlegmone des lk. Kniegelenks. 24 Std. nach Operation erneute Blutkulturen, die reichl. Bakterien enthielten.

8. XII. Erneute Schüttelfröste und Verschlechterung des Allgemeinbefindens. Zeichen einer Blutinfektion. Urotropin 3,0 g i. v. an 2 folgenden Tagen.

I I. XII. Eröffnung des lk. Kniegelenks, das vereitert ist. 20. XII. Langdauerndes schweres Krankenlager, starke langdauernde Eiterung aus den Wunden. Kein Zeichen einer Blutinfektion mehr. Blutkulturen enthalten keines Bakterien mehr. lassen.

26. III. I92I. Völlig geheilt, mit beweglichem Kniegelenk ent-

F all 4. Fr. E. R., 44 J. Vor 7 Tagen erkrankt mit einem Furunkel im Nacken, der vor 5 Tagen vom Arzt inzidiert wurde. $\mathrm{Da}$ Verschlechterung des Allgemeinbefindens und Schüttelfröste eintraten, wurde vom Arzt vor 3 Tagen eine neue Inzision gemacht. Unter erneutem Temperaturanstieg entstand vor 2 Tagen ein AbszeB an der $\mathbf{r}$. Brustseite. Frau in schwerkrankem Zustand mit schwersten Symptomen einer allg. Blutinfektion eingeliefert. Hohe Temperaturen, Schüttelfröste. In den Blutkulturen wuchsen in $1 \mathrm{ccm}$ Blut 45 Kol. Staphylococc. aur. und Mikrococc. tetragenus.

25. X. Wegen des schlechten Allgemeinbefindens von Operation zunächst abgesehen. Urotropin i. v. am ersten Tag 3,o g, am folgenden $6, \mathrm{og}$ und am dritten $6, \mathrm{og}$.

28. X. Nach der letzten Injektion von 6,og leichtes Brennen beim Wasserlassen. Im Urin geringer Eiweißgehalt, unter 0,5 Prom. 
Issbach. Keine Formelemente im Sediment. Die subjektiven Beschwerden, sowie der Eiweißgehait des Urins waren nach 24 Std. ganz verschwunden. 29. X. Temp. völlig abgefallen, keine Schüttelfröste. Pat. fühlt sich ganz wohl. Erneute Blutkulturen zeigen in $1 \mathrm{ccm}$ Blut 2 Kol. Staphylococc., kein Tetragenus. Es bildeten sich während der Blutinfektion an den verschiedensten Körperstellen kleine, etwas harte, sehr schmerzhafte subkutane Abszesse. Lwei derseiben wurden eröffnet und enthielten Staphyl. pyog. aur. und Tetragenus. Mehrere andere Abszesse bildeten sich nach Rückgang der Blutinfektion spontan zurück. 27. XI. Völlig geheilt und gut erholt entlassen.

F all 5. W. U., 39 J. V-Phlegmone der rechten Hand und des $r$. Armes. Wochenlange schwerste Eiterung aus vielen Operationswunden an $\mathrm{lk}$. Hand und $\mathrm{lk}$. Arm, so daß bei dem immer schlechteren Allgemeinbefinden an die Amputation des Armes gedacht wird. Im Blut sehr reichlich Staphylokokken. Nach 3 maligen Urotropininjektionen von 3,0-6,0 und 6,og wesentliche Besserung des Allgemeinbefindens. Langsames Nachlassen der Eiterung. Als geheilt mit gut erhaltenem Arm und gut erholt entlassen.

Fall 6. W. B., 45 J. Hüftgelenksvereiterung und AbszeB am Oberschenkel nach alter Granatsplitterverletzung. Nach Hüftgelenksresektion traten unter Fieberanstieg und Schüttelfrösten am I6. Tage post operationem Zeichen einer allgemeinen Blutinfektion ein. In I ccm Blut $47 \mathrm{Kol}$. Staphylococc. pyog, aur. und Pneumokokken. Erhielt 5 Tage hintereinander je 4,og Urotropin i. v. Danach Temperaturabfall, gute Heilung der Wunden, weiterer fieberfreier Verlauf. Geheilt entlassen.

F all 7. G. H., 21 J. Infizierte $\mathrm{H}$ üftgelenkswunde rechts nach früherer Kriegsverletzung. Nach eingreifenden Operationen an Knochen und Weichteilen schweres Krankenlager mit starker Eiterung aus den Wunden. Hohe Temperaturen und dauernde Verschlechterung des Allgemeinbefindens. Blutuntersuchung ergibt zahlreiche Kol. von Streptococc. long. Darauf intravenöse Urotropintherapie an 5 folgenden Tagen, im ganzen erhält Pat. 30,0 g Urotropin. Danach Allgemeinbefinden gebessert. Am 5. Tage der Injektionen Blutuntersuchung, die keine Bakterien im Blut ergibt. Sekretion aus den Wunden geringer. Granulationsbildung frischer und lebhafter. Befindet sich noch mit fast verheilter Wunde im Genesungsstadium in Behandlung.

F a 11 8. H. v. G., 29 J. Schwere Phlegmone des ganzen linken Beines bis zum Lig: inguinale. Allgemeine Blutinfektion. Pat. erkrankte vor 7 Tagen ohne nachweisbare äußere Ursache mit hohem Fieber, Schüttelfrösten und Erbrechen. 24 Std. nach Beginn der $E_{r}$ krankung trat die hochgradige Schwellung und Schmerzhaftigkeit des $\mathrm{lk}$. Beines ein, die rasch zu enormen Abszessen im lk. Ober- und 
Unterschenkel führte. Die Gelenke waren nicht befallen. In den Blutkulturen massenhaft Streptococc. longus und Staphylococc. pyog. aur.

28. I. 1921. Operation: Eröffnung von sehr großen Abszessen im lk. Oberschenkel und in der $\mathrm{lk}$. Wade. Die Eiterung ist nicht vom Knochen ausgegangen. Im Wundeiter Streptococc. longus und Staphylokokken. Mischinfektion.

29. I. Erhielt die nächsten 8 Tage, da sich das Allgemeinbefinden immer mehr verschlechterte, große Dosen Urotropin i. v., im granzen $44, \mathrm{og}$ Urotropin.

7. II. Pat. hatte keine Schüttelfröste, Temp. dauernd um 38,5 Grad, fühlt sich subjektiv wohl. Jedoch traten im Verlauf der nächsten Tage an verschiedenen Körperstellen metastatische Herde auf, im rechten Kniegelenk, in mehreren Fingergelenken und im r. Sternoklavikular-Gelenk. Ein günstiger Einfluß der Urotropinbehandlung war nicht zu erkennen. Von einer Amputation des lk. Beines mußte wegen der Ausdehnung der Phlegmone bis oberhalb des Hüftgelenks und wegen des schlechten Allgemeinzustandes Abstand genommen werden. II. II. Exitus letalis.

Obduktion: Eine Eingangspforte für die schwere Infektion lieb sich nicht nachweisen. Es handelte sich wahrscheinlich um eine primäre Mischinfektion des Blutes (Strepto-Staphylomycose) mit lokaler Ansiedlung der hochvirulenten Erreger im linken Bein und raschem Erliegen des Körpers durch die Schwere der Infektion.

F all 9. G. K., I 5 J. Eitrige Coxitis dextra. Pat. erkrankte vor 7 Wochen an einer Phlegmone des r. Handrückens, die vom Arzt inzidiert wurde, und die noch nicht ganz ausgeheilt ist. Vor 6 Tagen traten dann bei der Arbeit akut heftige Schmerzen im r. Hüftgelenk auf mit hohen Temperaturen und Schüttelfrösten. Die Blutkulturen zeigten $35 \mathrm{Kol}$. hämolys. Staphylokokken in $1 \mathrm{ccm}$ Blut.

2I. XII. I920. Operation: Osteotomie des r. Hüftgelenks. $1 / 2 \mathrm{Std}$. nach der Operation ließen sich kulturell in $\mathrm{I} \mathrm{ccm}$ Blut nur ca $5 \mathrm{Kol}$. hämol. Staphylococc. nachweisen. Einige Tage nach der Operation Verschlechterung des Allgemeinbefindens und erheblicher Anstieg. des Bakteriengehaltes im Blut. Darauf 6 Tage hintereinander intravenöse Urotropinbehandlung von tägl. $6,0 \mathrm{~g}$, bis der Bakteriengehalt im Blut bis auf etwa $\mathbf{I} \mathrm{Kol}$. in I $\mathbf{~ c m}$ abgefallen war.

Zur Zeit befindet sich der Pat. auf dem Wege der Besserung. noch in der Klinik. Bakterien sind seitdem im Blut nicht mehr nachzuweisen gewesen.

F all ro. F. S., 37 J. Schwere Phlegmone des ganzen r. Beines, ausgehend von einem Furunkel an der Schienbeinkante. Am I4. Tage nach der Operation bei noch bestehender starker Eiterung aus den Wunden Temperaturanstieg, Schüttelfröste, auffällige Verschlechterung des Befindens. Im Blut waren reichlich Streptococc. Iong. nach- 
weisbar. Er erhielt an 3 Tagen hintereinander je 6,o g. Urotropin i. v. Danach Besserung des Befindens. Im Blut später keine Bakterien mehr nachweisbar, guter Heilungsverlauf. Wurde mit gut verheilten Wunden gesund entlassen.

Fall II. H. F., I6 J. Osteomyelitis tibiae dextrae acuta. Vor 7 'Tagen, nach einem unbedeutenden Stoß am r. Kniegelenk erkrankte Pat. mit starker Schwellung und Schmerzhaftigkeit des ganzen r. Beines. Seit 5 Tagen Schüttelfröste. Übelkeit, hohe Temperaturen. In den Blutkulturen vor der Operation in I ccm etwa $25 \mathrm{Kol}$. hämolys. Staphylococc.

I5. III. I92 I. Operation: Osteotomia tibiae dextrae. Kniegelenk wird nicht eröffnet.

17. III. Erneuter Temperaturanstieg. Wiederauftreten der Schüttelfröste, Verschlechterung des Allgemeinbefindens. Zeichen einer schweren Blutinfektion. In den Blutkulturen in $\mathrm{I} \mathrm{ccm}$ Blut durchschnittl. $45 \mathrm{Kol}$. hämolys. Staphylococc. Zeichen einer Metastase im linken Kniegelenk. Erhielt 5 Tage hintereinander Urotropin i.v., zuerst täglich 4,o g, später $8, \mathrm{Og}$. 2I. III. Injektionen gut vertragen. Kulturell in $\mathrm{I} \mathrm{ccm}$ Blut höchstens $3 \mathrm{Kol}$. Staphylokokken. Ganz bedeutende Besserung des Allgemeinbefindens.

22. III. Eitriger ErguB im lk. Kniegelenk, in dem bakteriologisch sich sehr stark Staphylococc. aur. nachweisen ließ. (I Öse des Punktats rief auf Agarplatte $45 \mathrm{Kol}$. Staphylococc. hervor.) Nach Absaugen des eitrigen Exsudats Injeltion von $20 \mathrm{ccm}$ Urotropin 40 proz. in das Gelenk an 2 aufeinander folgenden Tagen. 25. III. Die Vereiterung des $\mathrm{lk}$. Kniegelenks (Metastase) hat sich spontan vollständig zurückgebildet. I6. IV. Seitdem keine Metastasenbildung mehr, keine Symptome einer Blutinfektion. Im Blut keine Bakterien nachweisbar. Gutes Allgemeinbefinden. Befindet sich noch wegen der Wundheilung in Behandlung.

F all I2. G. D., 54 J. Schwere Achselhöhlen- und Rückenhautphlegmone, verursacht durch Handverletzung an einem verrosteten Blechschornstein. 5 Tage nach der Operation unter hohem Temperaturanstieg Verschlechterung des Allgemeinbefindens, Schüttelfröste, Erbrechen. Blutinfektion. Im Wundeiter und im Blut fanden sich nur Streptokokken. Nach 3 maligen Injektionen von je 4,og Urotropin i. $v$, bedeutende Besserung. Rasches Abklingen der lokalen Eiterung und völiiges Verschwinden der Symptome der Blutinfektion. Völlig geheilt entlassen.

Fall I3. F. T., I4 J. Ostcomyelitis der ganzen rechten Tibia mit Beteiligung des r. Fußgelenks. Monatelanges Krankenlager mit nicht zu bekämpfender Eiterung aus den Operationswunden. Dauernde Verschlechterung des Allgemeinbefindens. Bei wiederholten Blutuntersuchungen fanden sich konstant einige Staphylokokken-Kolonien in I $\mathrm{ccm}$ Blut. Es handelte sich um eine sehr chronisch verlaufende, 
den Pat. äußerst schädigende Blutinfektion. Nach 3 intravenösen Urotropininjektionen von je $4, \circ \mathrm{g}$ trat eine gut zu beobachtende Besserung sowohl im Allgemeinbefinden, wie auch in der Wundheilung ein. In späteren Blutkulturen waren keine pyogenen Keime mehr nachweisbar. Mit vollkommen verheilten Wunden und gesund entlassen.

F a 11 I4. W. F., 30 J. Kniegelenksvereiterung links und schwere parartikuläre Phlegmone des linken Beins. Vor II Tagen hatte sich Pat. mit einem Zugmesser tief in das linke Kniegelenk geschnitten. Der behandelnde Arzt hatte die durchschnittene Sehne (Lig. patellae) und die Haut genäht. Nach 2 Tagen schwoll das ganze linke Bein unter hohem Fieber an. Schüttelfröste, Erbrechen. Bei Einlieferung in die Klinik hohe Temperațur, sehr starke Schwellung und Schmerzhaftigkeit des ganzen linken Beins, Schüttelfröste, Übelkeit. Operation: Eröffnung des linken Kniegelenks und umfangreiche Spaltungen der Phlegmone. Nach Operation rapide Verschlechterung des Allgemeinzustandes. Rascher Temperaturanstieg auf 4I Grad. Zahlreiche Schüttelfröste. Im Blut zahlreiche Kol. Staphylococc. aur. und einzelne Streptococc. long. hämolyt. Schwere pyogene Blutinfektion. Trotz des sehr schlechten Allgemeinzustandes entschloß man sich doch zur Amputatio femoris und sofortiger energischer intravenöser Urotropinbehandlung. Er erhielt 4 Tage hintereinander je 4,o g Urotropin i.v. Darauf rasche Besserung des Allgemeinbefindens. Sofortiges Aufhören der Schüttelfröste und Abfall der Temperatur. In späteren Blutkulturen waren niemals mehr pyogene Erreger nachweisbar. Der Amputationsstumpf eiterte noch einige Zeit sehr stark, hat sich dann gut geschlossen. Eine erneute Blutinfektion trat nicht mehr auf.

Fall 15. E. A., 20 J. Osteomyelitis acuta fem. sinist. Nach Osteotomie und breiter Eröffnung der Markhöhle im lk. Femur bestand wochenlange, nicht zu stillende Eiterung aus den Operationswunden und der Markhöhle am $1 \mathrm{k}$. Oberschenkel. Wiederholte bakteriologische Blutunteršuchung ergab konstant ein Vorhandensein von Staphylokokken-Kol. in $1 \mathrm{ccm}$ Blut. Nach 3 maligen intravenösen Urotropininjektionen von je 4,og an 3 folgenden Tagen beschleunigte sich die Heilung auffallend. Spätere Blutkulturen zeigten kein Staphylokokken-Wachstum mehr. Konnte nach einigen Wochen mit völlig geheilten Wunden entlassen werden.

Fal1 16. E. K., 19 J. Ausgedehnte beidseitige Mastitis pucrperalis nach infiziertem Abort. Schwere phlegmonöse Eiterung beider Brüste, die zu immer neuen Abszedierungen und Inzisionen führte. Konstant hohes Fieber, öfters Schüttelfröste. Allgemeinzustand verschlechterte sich immer mehr. Metastasenbildung in einem Kniegelenk. Im Blut wurden in 2 verschiedenen Untersuchungen jedesmal etwa 6 Kol. Streptococc. long. hämolyt. und mehrere Kol. Streptococc. brevis festgestellt. Nach 3 intravenösen Urotropininjektionen von 
je 4,o g trat eine sichtliche Verminderung der Sekretion aus den Wunden, völliges Verschwinden' der Schüttelfröste und besseres Allgemeinbefinden ein. Keine neuen Abszesse. Patientin konnte nach $3^{1} / 2$ Wochen mit gut verheilten Wunden gesund entlassen werden.

F all 17. W. H., $58 \mathrm{~J}$. Vor ungefähr 3 Wochen wurde Pat. von einem Pferde in den Ringfinger der lk. Hand gebissen, dabei wurde ihm vom Endglied des Fingers ein Stück Haut abgerissen. Am 3. Tage nach der Verletzung plötzlich Schüttelfrost und hohes Fieber, Erbrechen, Schmerzen in allen Gelenken. Die Schüttelfröste traten jeden Tag auf, auch mehrmals täglich. I. IV. I92I. Bei der Aufnahme war der Mann sehr elend, hatte hohe Temperaturen, sehr häufige Schüttelfröste, Übelkeit. Leber druckempfindlich, nicht vergrößert. Gesichts- und Körperfarbe lehmfarben, ikterisch. Der ganze Ringfinger linker Hand blaurot verfärbt und stark schmerzhaft, aber eigentlich nicht eitrig entzündet. Sonst keine phlegmonöse Entzündung am lk. Arm. Dabei bestand ein schweres Vitium cordis (Mitralstenose und Mitralinsuffiziens), das Pat. schon seit seinem I 5. Lebensjahr hatte. In den Blutkulturen wuchsen sehr reichlich hämolytische Staphylokokken.

4. IV. Morgens schweren Schüttelfrost, ebenso am Vormittag. Temp. auf 39,8 Grad gestiegen. Urinmenge sehr gering. Erhielt mittags $8,0 \mathrm{~g}$ Urotropin i.v. 5. IV. Urinmenge auf das Doppelte vermehrt. Pat. fühlt sich viel wohler, hat keinen Schüttelfrost mehr gehabt. 8o, g Urotropin i.v. Ebenso am 6. und 7. IV.

8. IV. Keine Schüttelfröste mehr, Pat. klagt darüber, daß sich in den Morgenstunden leichtes Brennen beim Wasserlassen und ziehende Schmerzen in der Blasengegend eingestellt hätten. Auch kann er den Urin nicht mehr recht halten. Im Urin, der spärlich und hochgestellt ist, befindet sich Blut, Albumen, aber keine Formelemente. Die Formaldehydprobe im Urin ist positiv, die Urotropinprobe negativ. Die subjektiven Beschwerden sind nach 6 Std. abgeklungen. (Thermophor auf die Blase.) I I. IV. Fieberfrei und fühlt sich wohl. Im Blut sind keine Bakterien nachweisbar. Die Blasenbeschwerden sind ganz abgeklungen. Pat. fühlt sich bedeutend besser. Urin noch immer Spuren von Albumen. I3. IV. I Uhr mittags akute Herzschwäche, die durch reichliche Exzitantien nicht mehr zu behaben ist. Nach $\mathrm{I}^{1 / 2}$ Std. Exitus letalis unter schwerster Atemnot.

Obduktion: Sehr weites schlaffes Herz. Alte Mitralstenose und Mitralinsuffizienz, starke Hypertrophie des r. Ventrikels, dicke Fibrinauflagerungen auf den Mitralklappen. (Endocarditis lenta.) Keine Lungenveränderungen mit Ausnahme yon beiderseitigen mäßigen Hypostasen. Alter Milztumor, keine frischen Milzabszesse. Keine akuten Nierenschädigungen. Auf der Blasenschleimhaut zahlreiche punktförmige Kapillarblutungen, kein Eiter. Sonst keine Metastasen. Tod bedingt durch alten Herzfehler. 
F all 18. B. R., 44 J. Die etwas indolente Patientin war vor I Woche mit einem Gesichtskarbunkel erkrankt, der die ganze $r$. Wange einnahm, so da $B$ Mund und $r$. Auge vollkommen verschwollen waren. Der Karbunkel wurde sogleich nach der Aufnahme in Äthernarkose bis ins Gesunde exzidiert. Am 3. Tage nach der Operation erkrankte die Pat. mit Schüttelfrösten, Temperaturanstiegen, Übelkeit, elendem Allgemeinbefinden. In den Blutkulturen fanden sich sehr reichlich Staphylokokken. Am 8. Tage trat eine Metastase in der $\mathrm{lk}$. Brust auf, die sich die Pat. wahrscheinlich beim Liegen während der I. Operation gedrückt hatte. Nach 4 Urotropininjektionen von je 8,og intravenös an 4 folgenden Tagen schwanden die schweren Allgemeinerscheinungen vollständig, in den späteren Blutkulturen fanden sich nur noch einzelne Bakterien. Später bildete sich noch eine Metastase in der Gegend der r. Clavicula, vielleicht durch Druck des Brustdrüsenverbandes bedingt, diese kam aber nicht mehr zur Vereiterung. Am 7. Tage nach der Urotropinbehandlung befand sich die Pat, völlig fieberfrei und wohl, stand auf und hat seitdem keine neue Blutinfektion erlitten. Geheilt.

Bei kritischer Beurteilung der vorher beschriebenen Krankheitsfälle muß zunächst einiges über den Charakter des Krankenmaterials gesagt werden. Es handelt sich bei unseren Fällen ausschließlich um pyogene Blutinfektionen nach chirurgischen Erkrankungen. Fast immer sind es sekundäre Blutinfektionen im AnschluB an Phlegmonen, Nackenfurunkeln, Osteomyelitis usw. Gar nicht zur Behandlung kamen die meist sehr schweren Blutinfektionen nach Erkrankungen im Bereich der weiblichen Genitalien, ferner diejenigen nach infektiösen Darmerkrankungen ( $T y$ phus, Dysenterie usw.) und die nicht minder schweren Blutinfektionen im frühesten Kindesalter. Es lassen sich also aus der sehr geringen Anzahl von Fällen und dem dazu noch sehr eingeschränkten Material tatsächlich wenig praktische Schlüsse ziehen. Zahlenangaben aus so geringem Material haben wenig praktischen Wert. Wir hatten unter 18 Fällen von pyogener Blutinfektion, die mit Urotropin intravenös behandelt worden sind, 2 Todesfälle, also I I Proz. Mortalität. Im Falle 17 ist der schließliche Exitus letalis, wie die Obduktion einwandfrei ergeben hat, nicht durch die pyogene Blutinfektion bedingt gewesen, sondern durch den seit Jahrzehnten bestehenden schweren Herzfehler. Auch ergab die Obduktion, daß es sich in diesem Fall um eine äußerst chronisch verlaufende Form der pyogenen Blutinfektion handelte, die bereits zu schweren Veränderungen der Herzklappen geführt hatte 
(Endocarditis lenta-Schottmüller) und die durch ein späteres verhältnismäßig geringfügiges Trauma zum plötzlichen Wiederaufflammen gebracht worden war. Bei derartigen chronisch verlaufenden Blutinfektionen mit schon bestehenden schweren Schädigungen der Herzklappen ist, wie seit langem bekannt, jede spezifische Therapie aussichtslos. Im Falle 2 handelte es sich um ein Kind, das in elendem Zustand, sehr spät, mit schon bestehenden beiderseitigen metastatischen Lungenabszessen zur Operation und zur Behandlung der Blutinfektion gelangte. Erschwerend kam hinzu, daß es sich um eine Infektion mit hämolysierendem Streptococcus longus handelte. Trotzdem gelang es, das sehr elende Kind noch über 2 Wochen zu halten und die schweren Symptome der Blutinfektion ganz aus dem Krankheitsbilde zu entfernen, bis der Tod hauptsächlich durch die ausgedehnten metastatischen Lungenveränderungen herbeigeführt wurde. Eine schwere pyogene Mischinfektion war Fall 8. Mischinfektionen sind überhaupt prognostisch sehr viel ungünstiger und besonders, wenn die enorme lokale Eiterquelle, wie in unserem Fall 8, auf chirurgischem Wege nicht vollständig ausgeschaltet werden konnte. Wenn es sich auch in diesen beiden Fällen um schwerste, eigentlich hoffnungslose pyogene Blutinfektionen handelte, so habe ich mich trotzdem auch hier nicht gescheut, mit Urotropin intravenös zu behandeln und den Mißerfolg zu bekennen; denn derartige hoffnungslose Fälle können wohl ein neues, bisher nicht verwendetes Therapeuticum leicht mißkreditieren, andererseits bedeuten sie aber den besten Wertmesser für die therapeutische Spannkraft und Elastizität eines Mittels, das in minderschweren Fällen als gut erkannt ist. Auch bei den geheilten pyogenen Blutinfektionen waren schwere Fälle (Fall I, 4 und 5), bei denen die intravenöse Urotropinbehandlung einen zweifellosen therapeutischen Erfolg bedeutete.

\section{Zusammenfassung.}

Bei der auch heute noch prognostisch sehr ernsten Krankheit der pyogenen Blutinfektion und der Unsicherheit sowohl in der Anwendung wie in der Wirkung der heute üblichen kausalen Mittel kann ein Versuch mit einem neuartigen Therapeuticum nicht ganz verworfen werden, besonders da dasselbe 
durch zahlreiche klinische Beobachtungen als relativ unschädlich in der Blutbahn bekannt ist. Es bedeutet vielleicht keinen Fortschritt in der Behandlung der pyogenen Blutinfektion, aber doch ein wertvolles Hilfsmittel, das natürlich erst durch längere Erfahrung erprobt werden muß. Nach objektiven Tatsachen, die ich an einer geringen Anzahl von Krankheitsfällen und sehr eingeschränktem Material gesehen habe, glaube ich, zu einem Versuch mit intravenösen Urotropininjektionen bei pyogener Blutinfektion raten zu dürfen, solange es auf diesem Gebiet keine wirklich zuverlässige und erfolgsichere andere Behandlungsmethode gibt.

\section{Literaturübersicht.}

1. B ertelsmann. Deutsche Zeitschr. f. klin. Chir., 72.

2. Blumenthal. Deutsche med. Wochenschr. IgIo.

3. -, Ther. d. Geg. IgII.

4. Boni. L'hosp. maj. Mailand 1913.

5. B ondy, P a yr-Küt tner. Erg. d. Chir. u. Orth, 7.

6. B rawermann. Diss. Leipzig I 909.

7. Brunner. Korr. f. schweiz. Ärzte I9Io.

8. Bubarew. Prakt. Wratsch 1913.

9. Crowe. John H. Baltimor Igog.

10. D anve. Gaz. d. hop. I906.

II. De ut $\mathrm{c}$ h. Wiener klin. Wochenschr. 1919, Nr. 45.

12. Ehrlich. Zeitschr, f. ärztl. Fortb. 1909.

13. Graff, v. Beitr. z. klin. Chir., Nr. 59.

14. GroB. Wiener klin. Wochenschr. 19r4, Nr. 22.

15. Hanzlik. Cleveland med. Journ. 1914, Nr. I2.

16. H of b a u er. Arch. f. Gyn., 68.

17. $\mathrm{Hug} \mathrm{g}$ ins. Amer. journ. of obst. I912.

I8. Jochmann, Lubarsch-Ostertag. Erg. d. Path., Io.

19. -. Zeitschr. f. Hyg., 6r.

2o. Kiraleyfi. Zeitschr. f. klin. Med., 68.

2I. Koch. Erg. d. Path. I909.

22. Kollarczek. Handb. d. Ver. Ther. München rgro.

23. Küstner. Med. Klinik rgo6.

24. Le nharts, Die sept. Erkrankungen. Wien 1903.

25. Lexer. Zeitschr. f. ärztl. Fortb. 1906.

26. Loebis ch. Wiener med. Presse 1901, Nr. 27 u. 28.

27. Loeper n. GroBdidier. Prog. med. 1918, Nr. 35, 36 u. 51.

28. Mikulicz u. Miyake. Deutsch. Chir.Kongr. 1904.

29. Mi y ake. Mitt. a. d. Grenzgeb. d. Med. u. Chir., I3.

30. Morgenroth. Berliner klin. Wochenschr. 19I I, 34 u. 44 . 
go Buzello, Über die Behandlung der pyogenen Blutinfektion usw.

31. Nic olaier. Zeitschr. f. klin. Med. I899, Nr. 38.

32. Ohira. Zentralbl. f. Bakt. Org. 1920, 85.

33. Patschkowski. Ther. Monatsh., Dez. 1904.

34. Sa chs. Wiener klin. Wochenschr. I916, Nr. 32.

35. - Wiener klin. Wochenschr. 1919, Nr. 24.

36. - Wiener klin. Wochenschr. 1919, Nr. 51 .

37. S passokukotzki. Mitt. a. d. Grenzgeb., Nr. 20.

38. Tavel. Deutsche med. Wochenschr. 1903.

39. W eil. Zeitschr. f. Hyg. rgi I.

40. W e it 1 a n er. Klin. ther. Wochenschr. 1908, Nr. 36.

4I. Wr e de. Arch. f. klin. Chir. 84.

42. $\mathrm{Z}$ a ngem e ister. Münchner med. Wochenschr. 1907 .

43. - Münchner med. Wochenschr. Igro. 